\section{Some Binding Properties of Human Serum Albumin as Studied by the Spin Labels 12-Doxyl Stearic Acid and its Methyl Ester}

\section{CARL LAGERCRANTZ and MORIO SETAKA*}

Department of Medical Physics, University of Göteborg, S-400 33 Göteborg 33, Sweden

Serum albumin has the ability to bind a large variety of substances in complexes which involve non-covalent bonds. By this means long chain fatty acids, bilirubin, and many drugs, otherwise of a very low solubility in the water phase, are dissolved and transported in the blood.

The study of this subject is of importance from several points of view. Thus the binding ability of serum albumin is a unique property of this protein. Further, the competition for binding sites between different substances can produce toxic concentrations of substances such as bilirubin and certain drugs when released from the serum albumin. Numerous studies have been devoted to these problems, mainly using ultrafiltration and equilibrium dialysis. ${ }^{1}$

The binding of a nitroxide spin label 2 to a large slowly rotating molecule such as serum albumin is expected to produce a considerable deviation from the sharp three-line ESR spectrum characteristic for the unbound spin label, and a complete immobilization relative to the serum albumin molecule is expected to produce a powder spectrum. The spin label method has so far been used for studying the motional and conformational properties of serum albumin after the attachment of the spin label through a covalent bond. ${ }^{3}$ Spin labeling of biological membranes has been performed by the use of serum albumin as a carrier of spin labeled fatty acids of low solubility in the water phase.4 In connection with the latter studies it was observed that spin labeled fatty acids gave rise to a powder spectrum when attached to serum albumin.

In this note it is demonstrated that the anisotropic properties of stable nitroxide radicals complexed with serum albumin can be very conveniently utilized for the study of the binding and competition of various low-weight molecular substances to serum albumin in addition to ultrafiltration and equilibrium dialysis.

In the present study some preliminary results are described which were obtained by the use of the stable nitroxide spin label 12-doxyl stearic acid (the $N$-oxyl-4', $4^{\prime}$-dimethyloxazolidine derivative of 12-oxostearic acid) and the

* On leave from the Faculty of Pharmaceutical Sciences, University of Tokyo, Bunkyo-ku, Tokyo, Japan. corresponding methyl ester. The experiments were performed with a $0.5,2$, or $5 \%$ solution of human serum albumin (HSA) in $0.2 \mathrm{M}$ phosphate buffer pH 7.4, a pH at which the carboxylic group of 12-doxyl stearic acid is completely dissociated. The concentration of the spin label was between $0.1 \times 10^{-6}$ and $2.0 \times 10^{-6} \mathrm{~mol} / \mathrm{ml}$ sample solution. Some experiments were also performed with the Cohn fractions IV and II, roughly corresponding to $\alpha$ - and $\beta$-globulins, respectively, and with human $\gamma$-globulin.

$A$. $H S A$. The experimental results are described for 12-doxyl stearic acid-HSA as follows, but were essentially identical with those obtained with its methyl ester.

(1) 12-Doxyl steric acid-HSA gave rise to a powder spectrum with no trace of a sharp three-line spectrum. However, when the relative concentration of the spin label was increased so that the molar ratio of the spin label to HSA exceeded a value of about 2, a superposition of the sharp three-line spectrum just appeared (Fig. I A), which increased upon a further increase of the relative concentration of the spin label.

(2) Treatment of the system 12-doxyl stearic acid - HSA exhibiting a pure powder spectrum with active charcoal as described by Chen for removing bound fatty acids from serum albumin, reduced the amount of bound spin label to about $1-2 \%$ of the original value.
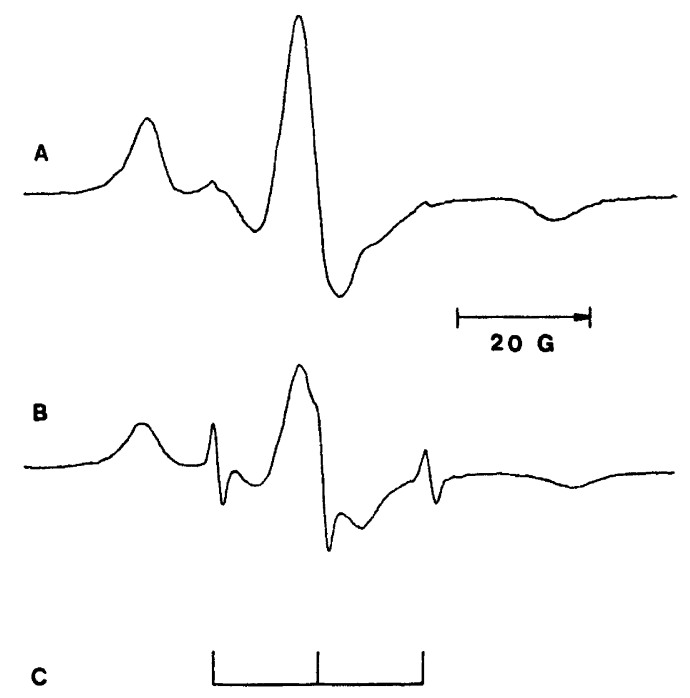

Fig. 1. ESR spectrum of the spin label 12-doxyl stearic acid in a $2 \%$ solution of human serum albumin (HSA), 0.2 M phosphate buffer pH 7.4. $A$. Molar ratio spin label/HSA $\sim 1.7$. $B$. After heating of $A$ at $80^{\circ} \mathrm{C}$ for $10 \mathrm{~min}$. $C$. Stick diagram of the sharp three-line spectrum. 
(3) Heating a solution of 12-doxyl stearic acid-HSA, which originally exhibited only the powder spectrum, at $80^{\circ} \mathrm{C}$ for 5 to $10 \mathrm{~min}$, brought about the appearance of the sharp three-line spectrum accompanied by a decrease of the powder spectrum (Fig. 1 B). The same result was obtained when the solution of HSA was heated prior to the addition of the spin label.

(4) After the digestion of HSA by pepsin at pH 3 for $3 \mathrm{~h}$, the ESR spectrum exhibited a superposition of powder spectrum and sharp three-line spectrum when 12-doxyl stearic acid was added to the reaction mixture so that the molar ratio of the spin label to HSA was 2.

(5) Competition experiments were performed with substances such as sodium benzoate, sodium salicylate, and sodium decanoate. When these substances were added in excess to the system of 12-doxyl stearic acid - HSA originally exhibiting only a powder spectrum, the sharp three-line spectrum appeared. An increase of the sharp three-line spectrum was always accompanied by a decrease of the powder spectrum. A similar result was obtained after the addition of ethanol to a final concentration of about $10 \%$.

$B$. $\alpha$ - and $\beta$-Globulins. 12-Doxyl stearic acid and its methyl ester respectively, gave rise to overlaps of sharp three-line spectra and powder spectra for all concentrations investigated, and it was not possible to remove the sharp threeline spectra by decreasing the concentration of the spin label relative to the $\alpha$ - or $\beta$-globulin, or by ultrafiltration of the solution, a separation which could be accomplished for the system 12-doxyl stearic acid - HSA.

C. $y$-Globulin. Only a very small amount of powder spectrum could be detected in the system 12-doxyl stearic acid (or its methyl ester) - $\gamma$-globulin.

The powder spectra indicated a complete immobilization of 12-doxyl stearic acid relative to the HSA molecule. This would mean that the spin label is attached not only by means of the dissociated carboxylic group at the end of the molecule, but that it is also attached in the whole of its length to the HSA molecule, or at least by means of the oxazolidine ring carrying the free radical function. The almost identical result obtained with the methyl ester of 12doxyl stearic acid seems to reduce further the importance of the dissociated carboxylic group for the attachment of 12-doxyl stearic acid to HSA.

The finding that about 2 molecules of 12 . doxyl stearic acid could be firmly bound to each HSA molecule is in agreement with the number of strong binding sites for fatty acids established by equilibrium dialysis." The de. crease of the powder spectrum followed by an increase of the sharp three-line spectrum upon heating, or digestion by pepsin, reflects the loss of binding sites when the protein was denaturated or partially split, respectively.
The competition experiments showed that 12-doxyl stearic acid could be displaced from HSA by an excess of substances such as decanoate and salicylic acid. Experiments are in progress for utilizing the competition between a spin label and a variety of low-weight molecular substances as a quantitative method for the evaluation of their relative binding ability to HSA.

The binding of 12-doxyl stearic acid and its methyl ester to $\alpha$ - and $\beta$-globulins seems to be much weaker than to H.SA. It is possible that the binding sites are localized to the lipid part of these globulins.

The very low binding of 12-doxyl stearic acid to $\gamma$-globulin seems to be in agreement with the known binding properties of this globulin when no hapten - antibody relationship is present.

Acknowledgement. We are indebted to Mrs. Th. Larsson for technical assistance. This work was supported by grants from The Swedish Natural Science Research Council.

1. Foster, J. F. In Putnam, F. W., Ed., The Plasma Proteins, Academic, New York 1960, Vol. 1, p. 178; Putnam, F. W. In Neurath, H., Ed., The proteins, Academic, New York 1963, Vol. III, p. 187; Peters, Jr., T. Advan. Clin. Chem. 13 (1970) 37.

2. Jost, P., Waggoner, A. S. and Griffith, O. H. In Rothfield, L. I., Ed., Structure and Function of Biological Membranes, Academic, New York 1971, Chapter 3; Smith, I. C. Biological Applications of Electron Spin Resonance, Wiley-Interscience, New York 1972, Chapter 11.

3. Stone, T. J., Buckman, T., Nordio, P. L. and McConnell, H. M. Proc. Nat. Acad. Sci. U.S. 54 (1965) 1010.

4. Keith, A., Bulfield, G. and Snipes, W. Biophys. J. 10 (1970) 618.

5. Chen, R. F. J. Biol. Chem. 242 (1967) 173.

6. Andersson, L.-O., Brandt, J. and Johansson, S. Arch. Biochem. Biophys. 146 (1971) 428.

Received December 23, 1974. 